\title{
Signal characterisation and processing in the forward scatter mode of bistatic passive coherent location systems
}

\author{
Marina Gashinova ${ }^{1 *}$, Liam Daniel ${ }^{1}$, Edward Hoare ${ }^{1}$, Vladimir Sizov² ${ }^{2}$ Kalin Kabakchiev ${ }^{1}$ and Mikhail Cherniakov ${ }^{1}$
}

\begin{abstract}
The transfer of the forward scatter (FS) concept to passive coherent location (FS PCL) systems provides a new emerging area of research. This article is dedicated to the investigation of various aspects of a bistatic passive coherent location (PCL) system operating in the FS mode. For efficient signal processing, appropriate FS PCL system analysis is presented. It is shown that using a relatively small modernisation of traditional signal processing algorithms, a PCL system may effectively operate against stealth and low profile targets crossing or being located in the vicinity of the radar baseline. The FS signals have been analysed in view of finding key effects and parameters influencing the waveforms and spectra which define the overall signal processing. Experimental results are given to validate the presented analysis.
\end{abstract}

Keywords: Passive coherent location (PCL), Transmitters of opportunity, Forward scatter, Self mixing receiver, Receiver phase noise, Coherent signal processing, Power budget

\section{Introduction}

Bistatic radars (BRs) have experienced resurgence in the radar community over the last decade, $[1,2]$ and the concept of passive coherent location (PCL) plays an important role in this new wave of interest.

The PCL systems form a specific class of BR which utilise emitters of opportunity to detect and track targets. Potentially any radio frequency emitter can be used as a non-cooperative transmitter for PCL [3] and Table 1 shows a selection of the available sources reported in the literature as PCL systems [4-14], operating from VHF to $K$ bands. The main operational mode for target detection and tracking in PCL is bistatic, where only the scattered signal from the target may be used for the extraction of range, trajectory information, and classification. In $[15,16]$, the main aspects of such a class of radar have been considered and it was touched upon, that if the extreme case of bistatic geometry can be exploited, the forward scatter (FS) operational mode

\footnotetext{
* Correspondence: m.s.gashinova@bham.ac.uk

${ }^{1}$ Electronic, Electrical \& Computer Engineering, University of Birmingham, Edgbaston, Birmingham B15 2TT, UK

Full list of author information is available at the end of the article
}

will potentially deliver significant improvement in "stealth" target detection.

There are both advantages and shortcomings of forward scatter radar (FSR). However, commonly shared opinion is that FSR has rather limiting capabilities, such as limited coverage due to narrow angular width of the main shadow lobe, strong clutter, presence of the Doppler dead zone, and, therefore, will not significantly contribute into the performance of PCL. In [17-23], however, it was shown that dedicated FSR can provide excellent Doppler resolution and its algorithms enable both estimation of target trajectory and speed and classification. This article aims to show that FSR when integrated into PCL systems will have the same capabilities as in the case of dedicated FSR, will add extra benefits to the existing bistatic mode of operation of PCL and can practically be implemented on both hardware and software levels without requiring significant restructuring.

FSR forms a sub-class/mode of BR, one where targets are observed at large bistatic angles $\beta \approx 140^{\circ}-180^{\circ}$. FSR signatures are formed in a specific way, which govern the signal processing algorithms used. Depending on the scenario, signatures are composed of varying contributions 
Table 1 Example emitters of opportunity for PCL systems

\begin{tabular}{|c|c|c|c|}
\hline Emitter & Frequency, $(\mathrm{GHz})$ & Wavelength, (m) & Functionality as $\mathrm{PCL}$ \\
\hline FM Radio $[4,5]$ & 0.1 & 3 & Medium to long range air target detection and tracking. \\
\hline Digital Audio Broadcasting (DAB) [3] & 0.2 & 1.5 & \\
\hline \multirow{2}{*}{$\begin{array}{l}\text { Digital Video Broadcasting - Terrestrial } \\
\text { (DVB-T) }[3,6]\end{array}$} & 0.4 & 0.75 & \\
\hline & 0.6 & 0.50 & \\
\hline LEO satellite communication [7] & 1.5 & 0.2 & \\
\hline \multirow[t]{2}{*}{ Mobile Cellular Radio (MCR) [8] } & 0.9 & 0.33 & \multirow{2}{*}{$\begin{array}{l}\text { Short to medium range air targets and local vicinity surface } \\
\text { target observations. }\end{array}$} \\
\hline & 1.8 & 0.16 & \\
\hline Local Area wireless Network (LAN) $[9,10]$ & 2.4 & 0.13 & $\begin{array}{l}\text { Indoor and close range security applications intended for human } \\
\text { and vehicle detection. }\end{array}$ \\
\hline \multirow{2}{*}{$\begin{array}{l}\text { Global Navigation Satellites Systems } \\
\text { (GNSS) }[11,12]\end{array}$} & 1.5 & 0.2 & \multirow[t]{5}{*}{ Hybridisation with SAR imaging. } \\
\hline & 1.2 & 0.23 & \\
\hline \multirow[t]{2}{*}{ Synthetic Aperture Radar (SAR) [13] } & 3.0 & 0.1 & \\
\hline & 10.0 & 0.03 & \\
\hline Satellite TV (SatTV) [14] & 14.0 & 0.02 & \\
\hline
\end{tabular}

of both FS shadowing of the direct path signal and bistatic scattering when the target is in the vicinity of the baseline $[19,24,25]$ - to note, in PCL systems the direct path is usually referred to as the transmitter-receiver leakage signal $[15,16]$ and in bistatic configuration is typically unwanted. This highlights an advantage of FSR: the ability to detect stealth targets through observing perturbations in the direct path signal. As another example, the FS effect significantly increases target radar cross section (RCS) in the forward direction, this is irrelevant to target shape and material at least within the optical, or Mie scattering regions. This increase however exists only over a narrow spatial region (the forward scatter main lobe-FSML), thus limiting the use of FSR to 'microwave fence' applications. However, using transmitters of opportunity a ubiquitous FSR network could be built thus widening the area of its applicability. It should be mentioned that performance of FSR mode does not depend on the particular PCL signal modulation scheme and, therefore will not influence FSR signal processing complexity, implying that any available transmitter of opportunity could be used for forward scatter concept to passive coherent location (FS PCL) systems.

With this in mind, the main aim of this articleis to explore the integration of an FSR channel/mode into the traditional PCL system to compliment the conventional BR approach. The layout of the article is as follows: Section 2 will present the general passive radar layout, highlighting the inclusion/integration of the FSR channel. Section 3 looks at aspects of target cross section in relation to scattering region and comparison of monostatic and bistatic RCS (MRCS, BRCS) to that of the forward scatter cross section (FSCS). Section 4 concentrates on range resolution and Doppler analysis, indicating the inherent low Doppler frequencies that are observed and can in fact be measured in FSR. Optimal signal processing is also discussed in this section. Section 5 introduces the practical realisation of the system, through discussion of power budget, noise and the concept of the self-mixing receiver. Section 6 provides conclusions, where appropriate, simulations and experimental results from our dedicated FSR studies are included.

\section{PCL system topology and overview}

The generic layout of a passive BR system is shown in Figure 1, which shows the bistatic topology and traditional hardware signal processing; the proposed FSR subsection is also now included. The transmitter of opportunity, Tx, emits a signal of carrier $f_{0}$ and bandwidth $\Delta f_{0}$ which is scattered by the target $\mathrm{Tg}$, situated at a range $R_{T}$ and $R_{R}$ from the transmitter and receiver, respectively. The scattered signal is received at $\mathrm{Rx}$, which has a baseline distance of $R_{0}$ from Tx. In the traditional bistatic processing scheme, the heterodyne channel, $\mathrm{Hx}$, is intended for synchronisation (Synch) of the transmitted and received signals at the receiver end and used as a reference to down convert received signals to baseband; quadrature components $I$ and $Q$ are then formed to provide coherent signal processing [26]. In this regime of the PCL system, the spatial distribution of the target scattering is specified by the BRCS $\sigma_{\mathrm{BR}}[27,28]$. As the bistatic angle $\beta$ increases, the FSML becomes more aligned with the baseline, indicating the transition to the FS regime and the utilisation of the additional FSR subsection nonlinear processing block.

In PCL, we assume that a transmitted signal always reaches the designated receiver and therefore $\mathrm{Tx}$ and $\mathrm{Rx}$ antennas could be viewed as omni-directional with clear line of sight; for VHF and UHF bands' antennas are physically nearly omni-directional in any case. The remaining variables in Figure 1 will be explained as required in the following sections. 


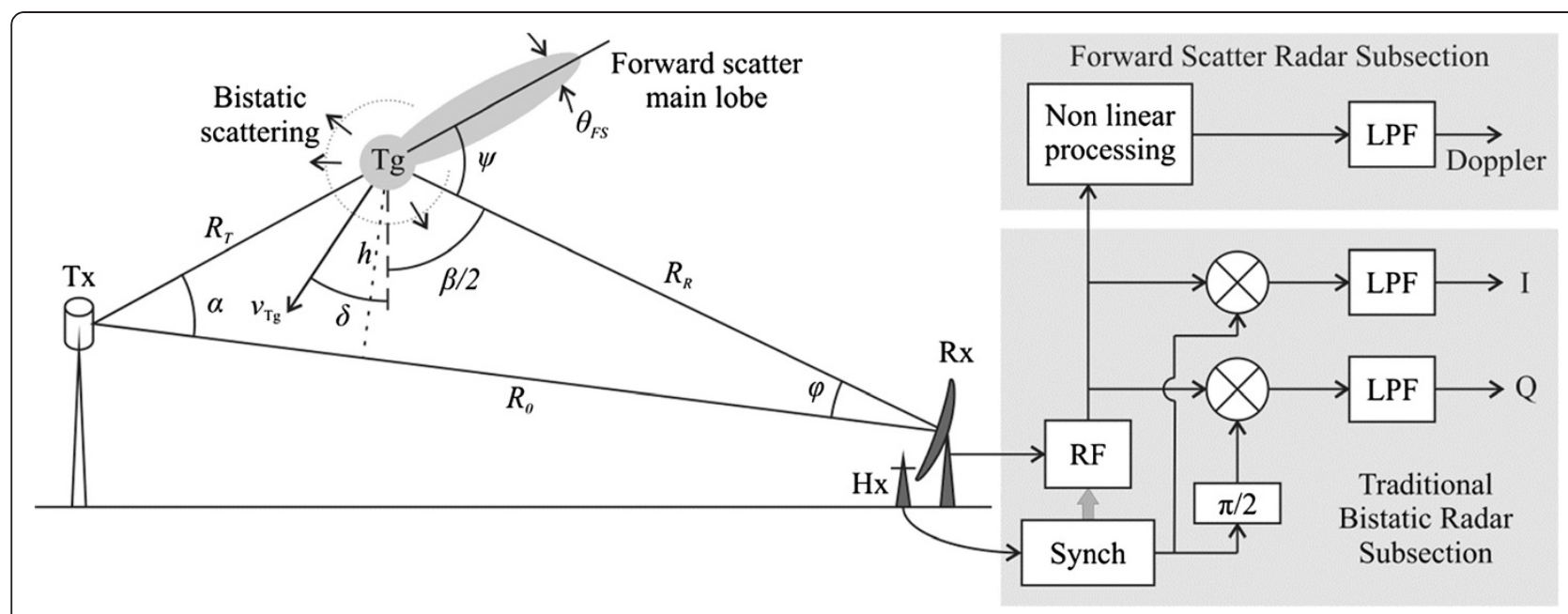

Figure 1 Generic BR topology and hardware signal processing, with added FS subsection.

\section{Target RCS}

An important benefit of using BR in an FS configuration is the enhancement in cross section, both in terms of magnitude and stability. In the following, there is a discussion of RCS in various scattering regimes, supported using the analytically known solution for the sphere. Consequently, EM simulations are provided for typical difficult targets to confirm in an exact way, the benefits of FSCS over BRCS in the appropriate scattering regimes.

\subsection{Phenomenology of FSCS}

All targets can be classified to fit into one of the known scattering regions; the Rayleigh region $(D / \lambda<<1)$, the resonance/Mie region $(D / \lambda \approx 1)$, or the optical region $(D / \lambda \gg>1)$, where $D$ is the typical target dimension. In the Rayleigh region, RCS is practically independent of bistatic angle; any noticeable increase in the FS direction is absent. In the Mie region, there is some increase in FSCS. In the optical region, irrelevant to the target nature, a significant enhancement of RCS in the FSML is always observed reaching the maximum value at bistatic angle $\beta=180^{\circ}$ [29,30], given by

$$
\sigma_{\mathrm{FSMax}}=4 \pi A^{2} / \lambda^{2},
$$

where $A$ is the physical target area and $\lambda$ the wavelength of the illuminating signal. This FSML is equivalent to the main lobe of an antenna with an aperture corresponding to the target silhouette [31,32]. Its width $\theta_{\mathrm{FS}}$ is estimated as

$$
\theta_{\mathrm{FS}} \approx K \lambda / D[\mathrm{rad}],
$$

where $K$ depends on the reference level of the FSML width and the actual target shape. Rectangular and spherical targets with FSML width defined at the $-3 \mathrm{~dB}$ level correspond to $K \approx 1$, but for more complex targets and/or a lower reference level, $K$ may be between 1 and 4. In practice, a simplified approach could be used $[19,28]$ whereby a target is approximated as a rectangular plate with length $L$ and height $H$. Hence, the $-3 \mathrm{~dB}$ level of FSML in azimuth plane will be $\theta_{\mathrm{FS}, \mathrm{az}} \approx \lambda / L$ and in elevation $\theta_{\mathrm{FS} \text {,el }} \approx \lambda / H$. A simplified definition can now be made, such that if the FSML is pointing to the radar receiver, the system is referred to as FSR, omitting any conditions on scattering region to provide enhanced RCS. Thus, the geometry in Figure 1 indicates that the radar will operate in the target FS region if

$$
\theta_{\mathrm{FS}} / 2 \geq \pi-\beta=\psi \quad \text { or } \quad \beta \geq \pi-\theta_{\mathrm{FS}} / 2 \approx \pi-\lambda /(2 D) .
$$

To demonstrate the fundamental difference between MRCS and FSCS the conductive sphere of diameter $D$ is analysed. In Figure 2, normalised values of MRCS and FSCS as well as FSML width $\theta_{\mathrm{FS}}$ are shown as functions of the normalised dimensionless parameter $p=\pi D / \lambda$. In contrast to the RCS which is restricted in the optical region by the physical geometric cross section, for FSR we can see a monotonic rise of the FSCS, indicating the trend of increasing gain from the FS effect. It should be stressed that for FS in the upper Mie and optical region, FSCS and $\theta_{\mathrm{FS}}$ (Equations 1 and 2) are related and for the sphere $(K=1)$,

$$
\begin{aligned}
\sigma_{\mathrm{FSMax}} & =\frac{\pi^{3}}{4} \cdot \frac{D^{2}}{\theta_{\mathrm{FS}}^{2}}=\pi^{2} \frac{A}{\theta_{\mathrm{FS}}^{2}} \quad \text { and } \\
\theta_{\mathrm{FS}} & =\pi \sqrt{A / \sigma_{\mathrm{Max}}} .
\end{aligned}
$$

Though derived for a sphere, this equation reflects the general relationship between cross section and the width of the main lobe for targets of any shape, i.e. the FSCS is 


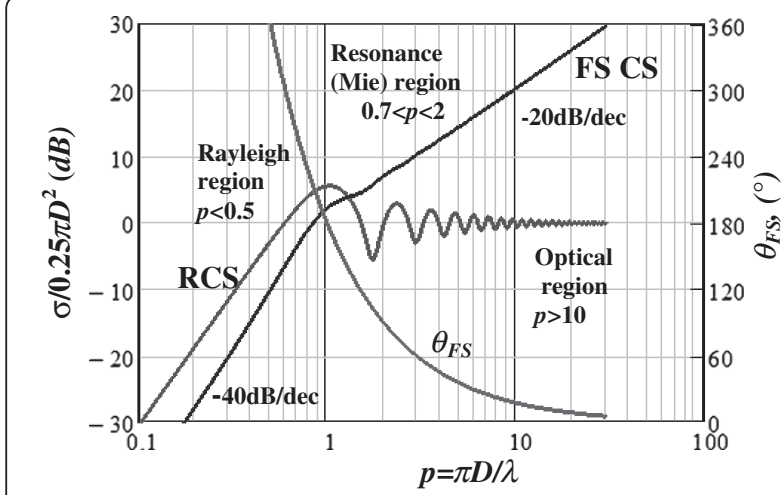

Figure 2 RCS and FSML width for spherical targets. Normalised values of MRCS and of FSCS as well as FSML width $\theta_{F S}\left({ }^{\circ}\right)$ are shown as the functions of the normalised dimensionless parameter $p=\pi \mathrm{D} / \lambda$.

inversely proportional to the square of the FSML width. We can therefore see (Figure 2) that the 'penalty' for the increase in target reflectivity is the narrowing of the FS region, e.g. for the sphere, a $10 \mathrm{~dB}$ increase in FSCS in the upper Mie region corresponds to a $40^{\circ} \mathrm{FSML}$ width reduction.

For comparison and analysis of more complex shapes to emphasise the conclusions drawn above, we must use 3-D full-wave simulation methods.

\subsection{Simulation of 3-D BRCS}

The BRCS of several objects of specific interest as representatives of 'difficult' targets (targets, for which detection is impeded due to their inherently low RCS and/or either very high or low speed) has been calculated in CST microwave studio [28] for available PCL signals. The simplified shapes of Figure 3 were made of perfectly electrically conducting (PEC) material and used in simulation. Results of the simulation of BRCS $\left(\beta=90^{\circ}\right)$ and of FSCS $\left(\beta=180^{\circ}\right)$ for broadside incidence of the electromagnetic wave are presented in Table 2. The target lengths $L$, heights $H$ and speeds $V$ are chosen as the most typical for each particular target. The chosen targets define scattering in all three regions-Rayleigh (R), Mie (M) and optical $(\mathrm{O})$-according to their dimensions and simulation carrier frequencies. This table demonstrates that for the Rayleigh region, there is no advantage in using FS mode in terms of RCS over the bistatic case. For the Mie region and, specifically, optical case however, the advantage is obvious. It should be noted that some of the very low BRCS values are due to the occurrence of nulls at $\beta=90^{\circ}$.

As a highlighted example the simulated RCS presented both in 3D and azimuth plane for the side illuminated missile are shown in Figures 4 and 5, respectively, for two wavelengths, defining the upper Rayleigh-low Mie (A) and optical scattering regions (B). For the $3.6-\mathrm{m}$

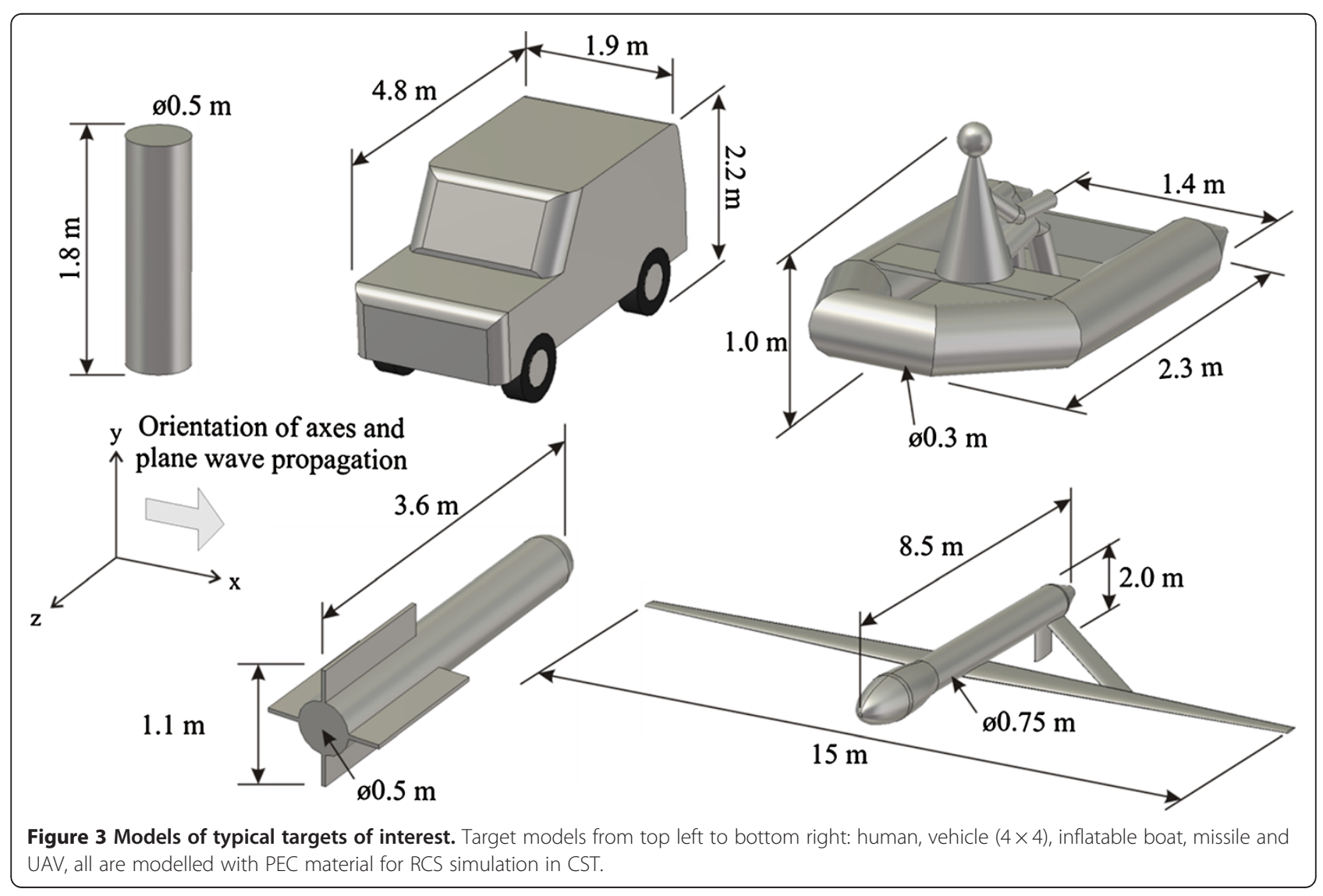


Table 2 Simulated BRCS and FSCS for a selection of targets in various scattering regimes

\begin{tabular}{|c|c|c|c|c|c|}
\hline & $\mathrm{RCS}, \mathrm{dBs}$ & & & & \\
\hline & $\mathrm{BR}(\beta=9$ & $\left.=180^{\circ}\right)$ & & & \\
\hline$\lambda \rightarrow$ & $3.0 \mathrm{~m}$ & $1.5 \mathrm{~m}$ & $0.75 \mathrm{~m}$ & $0.3 \mathrm{~m}$ & $0.1 \mathrm{~m}$ \\
\hline$f \rightarrow$ & $100 \mathrm{MHz}$ & $200 \mathrm{MHz}$ & $400 \mathrm{MHz}$ & $1 \mathrm{GHz}$ & $3 \mathrm{GHz}$ \\
\hline Targets, $L(\mathrm{~m}), H(\mathrm{~m}), V($ & & & & & \\
\hline Human & $6.2 / 7.6$ & 7.3/11.1 & 7.6/15.6 & $11.6 / 22.0$ & $15.4 / 33.2$ \\
\hline$L=0.5, H=1.8, V=1.0$ & (R) & (M) & $(\mathrm{M})$ & $(\mathrm{O})$ & (O) \\
\hline Vehicle, LR Discovery & $9.7 / 22.5$ & $7.6 / 28.2$ & $-7.4 / 34.1$ & $5.4 / 41.0$ & - \\
\hline$L=4.8, H=2.2, V=10$ & $(\mathrm{R} / \mathrm{M})$ & (M) & (sub-O) & $(\mathrm{O})$ & \\
\hline Inflatable boat & $0.6 / 2.7$ & $-1.3 / 6.8$ & $-9.4 / 13.0$ & $-6.6 / 21.4$ & $-7.3 / 40.3$ \\
\hline$L=2.3, H=1, V=5$ & (R) & (M) & (Sub-O) & $(\mathrm{O})$ & $(\mathrm{O})$ \\
\hline Missile & $2.9 / 8.9$ & 1.6/15.3 & $-8.0 / 22.6$ & $-6.1 / 29.0$ & $-3.2 / 45.0$ \\
\hline$L=3.6, H=0.5, V=200$ & (R/M) & (M) & (Sub-O) & $(\mathrm{O})$ & (O) \\
\hline UAV Predator & $4.4 / 17.6$ & $2.0 / 23.5$ & $-1.6 / 32.9$ & $-3.0 / 60.0$ & - \\
\hline$L=8.4, H=2, V=50$ & $(\mathrm{M})$ & (O) & (O) & (O) & \\
\hline
\end{tabular}

target length and the 3-m FM radio wavelength there are two well-defined maxima of back and forward scattering lobes: 11.7 and $9 \mathrm{dBsm}$, respectively. This indicates that in this low Mie region, back and forward scatterings are approximately the same intensity. Between these two rather wide forward and back scatter lobes, exist side scattering regions with on average around $8 \mathrm{~dB}$ less intensity than that for the maxima. For the same target, illuminated by a satellite TV signal of $0.3 \mathrm{~m}$ wavelength, there are also two pronounced maxima for back and forward scatterings. Now in the optical region however, both lobes are very narrow of a few degrees order, their maximum intensity is greater than in the FM radio case and the forward scattering peak is approximately $10 \mathrm{~dB}$ stronger than that of the back scattering. In the bistatic (or side) scattering direction, the RCS drops by up to $30-35 \mathrm{~dB}$ relative to the FS. Assuming that this missile is shaped as stealth for the monostatic radar (MR), the pronounced peak at $\beta \approx 0$ will be absent and the target is likely to be detected only in FS region.

\section{Range Resolution and Doppler in FSR}

Two perceived drawbacks of FSR are the loss of range resolution and the Doppler 'dead zone'. However, even though range resolution may be lost, the excellent Doppler resolution may partly compensate for this. It is also shown that in FSR the very low Doppler frequencies corresponding to the 'dead zone' (narrow FS lobe) can be measured and target Doppler signature can be used for detection.

\subsection{Range resolution}

Switching from bistatic to FSR introduces a reduction of the bistatic range resolution $\Delta R_{\mathrm{BR}}$ until its disappearance in the FSML region. The general equation for $\Delta R_{\mathrm{BR}}$ as a

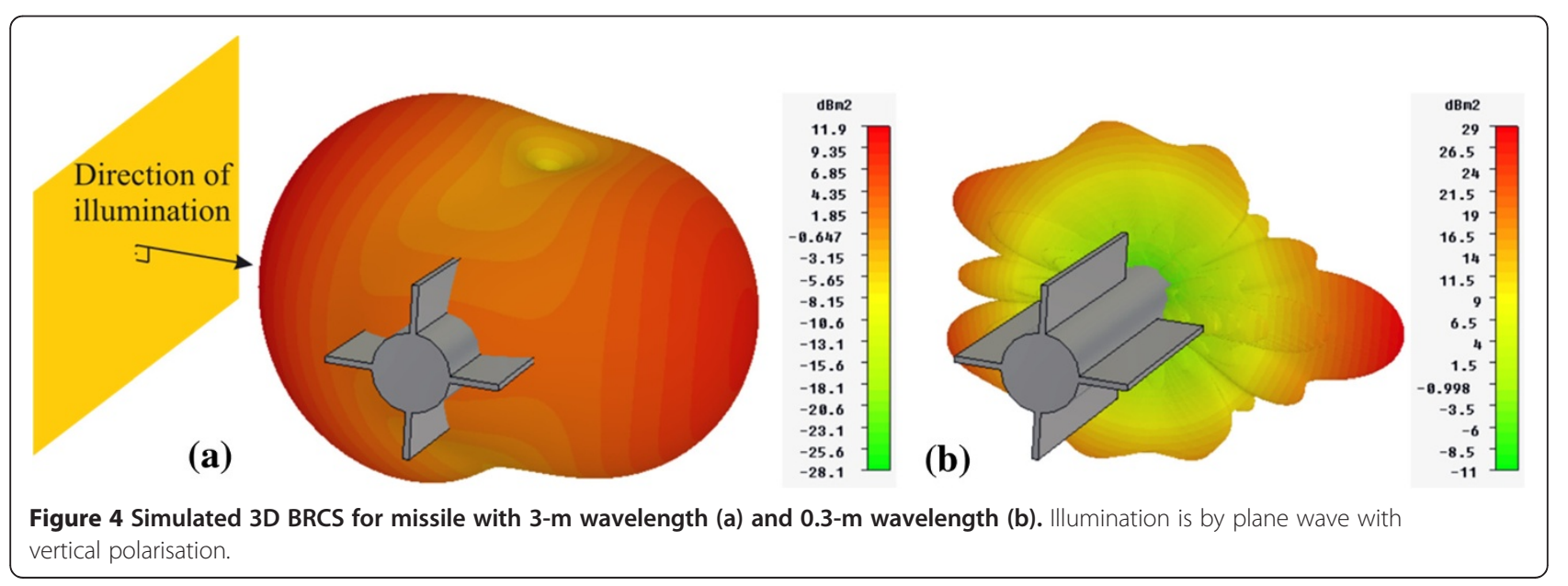




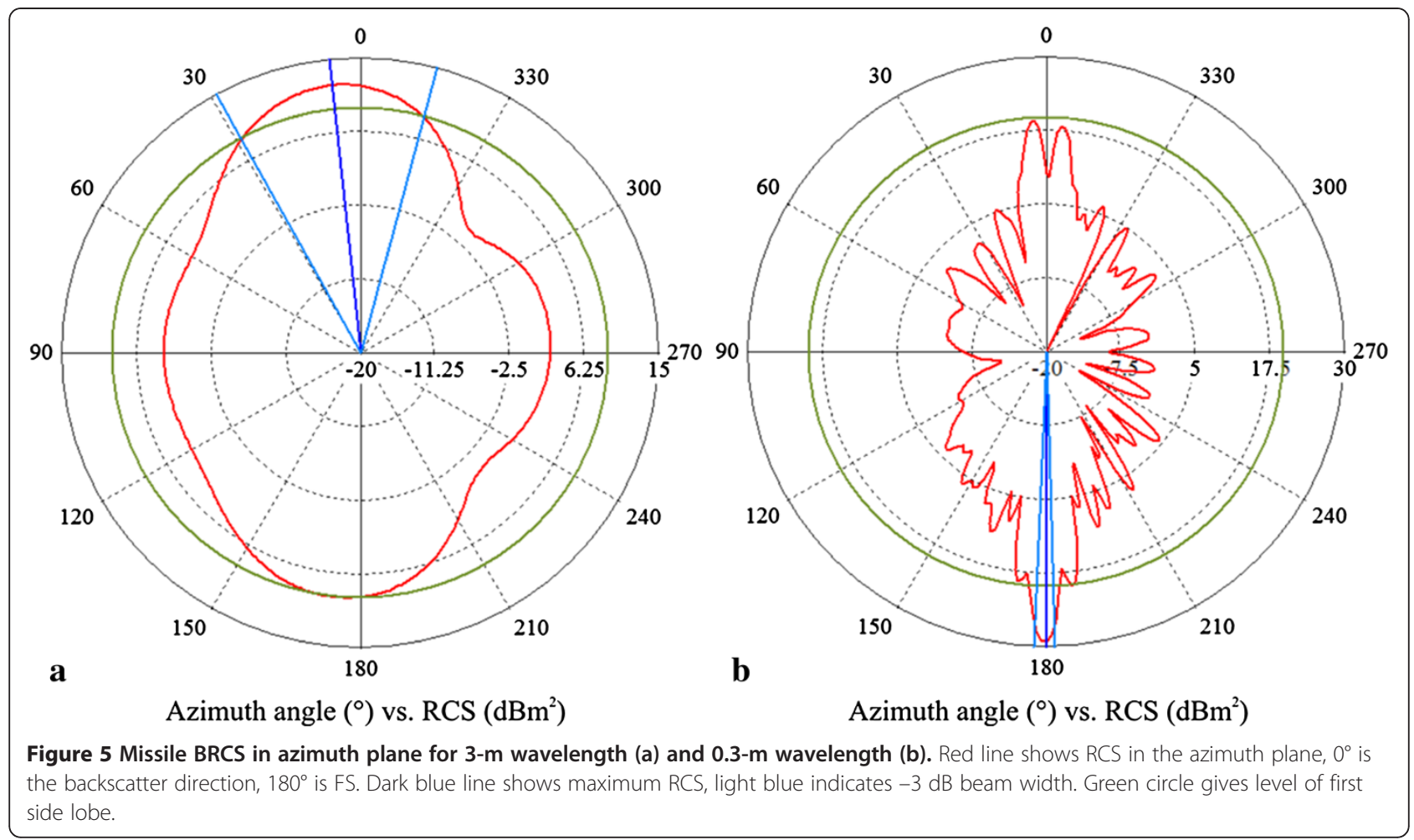

function of the bistatic angle $\beta$ and either the monostatic range resolution $\Delta R_{\mathrm{MR}}$ or the signal bandwidth $\Delta f_{0}$ is defined by the geometry in Figure 6 and can be expressed through [27],

$$
\Delta R_{\psi}=\frac{\Delta R_{\mathrm{MR}}}{\cos (\beta / 2) \cos \psi}=\frac{c}{2 \Delta f_{0} \cos (\beta / 2) \cos \psi} .
$$

In the bistatic configuration of Figure $6 \mathrm{a}, \mathrm{Tg} 3$ is spatially separated due to the receive antenna pattern. In the FS configuration (Figure 6b), $\mathrm{Tg} 3$ is spatially separated from the receiver even in the case of a wide beam receive antenna due to the narrow FSML. Range resolution in BR is specified along the bistatic bisector angle. If two targets$\operatorname{Tg} 1$ and $\operatorname{Tg} 2$ in Figure $6 \mathrm{a}$-are placed along this bisector $(\psi=0)$, the range resolution depends on the signal bandwidth $\Delta f_{0}$ and the bistatic angle $\beta$. If the second target is shifted relevant to the bisector (Figure 6a, $\mathrm{Tg} 3$ ), it experiences a resolution reduction factor of $\cos \psi$. If $\psi=90^{\circ}$, the two targets are on the same iso-range contour and are not distinguishable in
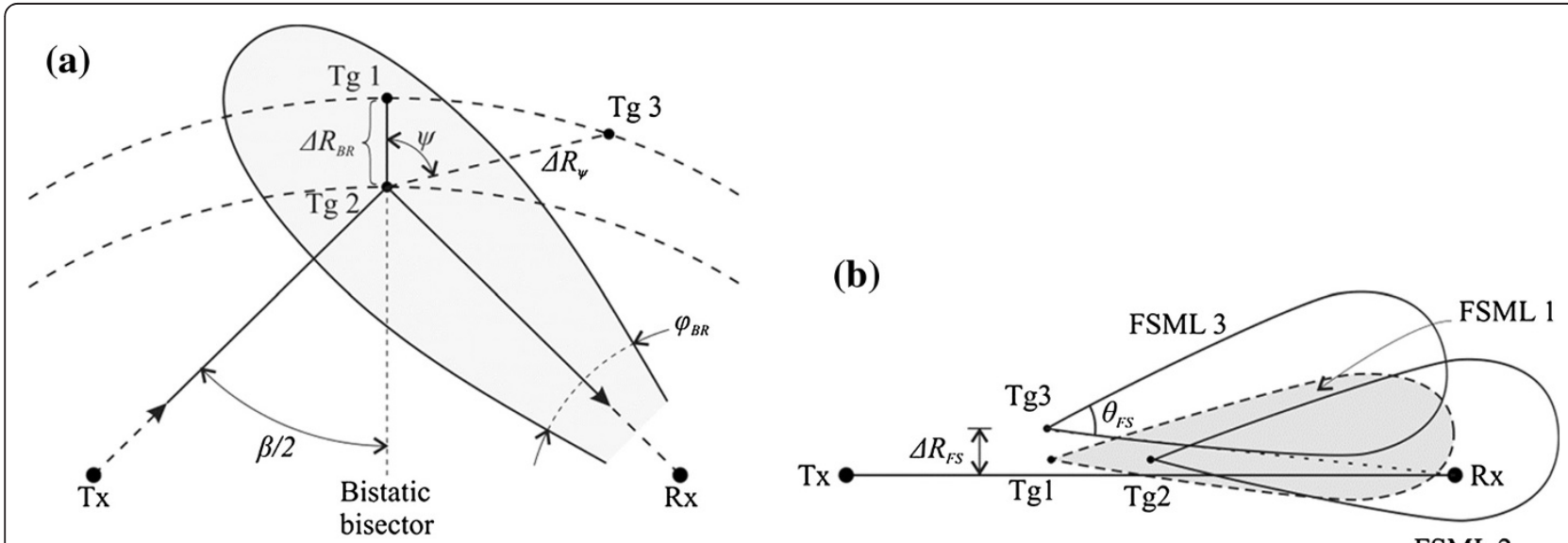

FSML 2

Figure 6 Range resolution in (A) bistatic and (B) FSR configurations. 
the time domain. In $\mathrm{BR}$, the range and angular resolution are coupled, potentially two targets at the same iso-range contour could be resolved if their receive antenna is directional. Assuming that the receive antenna beam width is $\phi_{\mathrm{BR}}$, the linear resolution of the two targets on the same iso-range contour could be estimated as $\Delta R \approx R_{R} \phi_{\mathrm{BR}}$ [rad], where $R_{R}$ is previously defined in Figure 1 as the target to receiver range. Equation (5) shows that under other equal conditions, the range resolution in $\mathrm{BR}$ is worse in comparison to MR.

As $\beta \rightarrow 180^{\circ}$ range resolution is dramatically reduced by a factor of $\cos (\beta / 2)$ and in the vicinity of the baseline, the range resolution direction is normal to the baseline. Evidently in contrast to BR, targets separated by the angle $\phi \rightarrow 90^{\circ}$ (Figure 6b, Tg1 and Tg2) are not resolved by means of time or angular resolution. When targets are aligned along the bistatic bisector (Figure 6b, Tg1 and $\mathrm{Tg} 3$ ), any practical resolution could be observed only in ultra-wideband (UWB) FSR [20], where extremely wide signal bandwidth $\Delta f_{0}$ 'compensates' the resolution reduction due to the very low value of $\cos (\beta / 2)$.

In FSR, there is one extra mechanism of target resolution placed along the bisector and can also be referred to as range resolution, but does not deal with signal separation due to a different delay (and hence does not relate to the signal spectrum $\Delta f_{0}$ ), nor is it reliant upon receive antenna directivity. There will be an angular resolution, where the narrow FSML is acting as a directional antenna. In Figure 6b, it is shown that the FSML from targets $\operatorname{Tg} 1$ and $\mathrm{Tg} 2$ is aimed towards the receive antenna and hence the FS signal is detected. The FSML of $\operatorname{Tg} 3$ is not pointed towards the receiver and hence Tg3 will be resolved from Tg1 and Tg2. This resolution, $\Delta R_{\mathrm{FS}}$, could be estimated as

$$
\Delta R_{\mathrm{FS}} \approx R_{R} \frac{\theta_{\mathrm{FS}}}{2},
$$

where $\theta_{\mathrm{FS}}$ is the FSML width. Using a rectangularshaped approximation of the target, $\theta_{\mathrm{FS}}=\lambda / h$, with $h$ being the target dimension in the appropriate plane and $\lambda$ being the illuminating wavelength, the resolution can be expressed as

$$
\Delta R_{\mathrm{FS}} \approx R_{R} \frac{\lambda}{2 h} \text {. }
$$

Or, the angular resolution of two targets form the receiver position will be given by, $\Delta \theta_{\mathrm{FS}}=\theta_{\mathrm{FS}} / 2=\lambda / 2 h$.

\subsection{Frequency resolution}

Whilst the absence of range resolution is an apparent drawback of FSR, it does however give rise to a nonfluctuating target signal, even for highly manoeuvrable targets. As a result, the maximum coherent analysis time in FSR may be equal to the target visibility time $T_{V}$. Thus, an absence of range resolution is partly compensated by the excellent frequency resolution.

We can now contrast two extreme scenarios of MR and FSR. In MR, the target fluctuation spectrum bandwidth $\Delta f_{M}$ and coherent analysis time $\Delta \tau_{M}$ are estimated as [33]:

$$
\Delta f_{M} \approx\left(\frac{D}{\lambda}\right)\left(\frac{\Delta \phi}{\Delta t}\right) \quad \text { and } \quad \Delta \tau_{M}=1 / \Delta f_{M} .
$$

where $D$ is an effective target dimension and $\Delta \phi / \Delta t$ is the rate of change of the aspect angle $\phi$. Effectively, $\Delta f_{M}$ corresponds to a frequency resolution limit in MR and $\Delta \tau_{M}$ is the maximum coherent integration time. Examples of the calculated fluctuation spectra bandwidth $\Delta f_{M}$ and maximum coherent integration time $\Delta \tau_{M}$ for UAV "Predator" (wingspan $D=12 \mathrm{~m}$ ) are presented in Table 3 for different wavelengths and aspect angle variation rates-it should be noted that the 3-m wavelength situation may not be expressed exactly by Equation (8) as it is not an optical case. Therefore, in MR the coherent analysis time $\Delta \tau_{M}$ is limited by the fluctuation spectra. In FSR, the target visibility time $\Delta \tau_{\mathrm{FS}}$ is equal to the target coherent analysis time. If a target moving with speed $v_{t g}$ is within the FSML its visibility time could be estimated as

$$
\Delta \tau_{\mathrm{FS}} \approx \frac{\lambda R_{0}}{2 D v_{t g}} .
$$

For comparison with the monostatic case, $\Delta \tau_{\mathrm{FS}}$ and the FSR frequency resolution $\Delta f_{\mathrm{FS}}=1 / \Delta \tau_{\mathrm{FS}}$ are shown in the last two columns of Table 3 for the UAV midpoint crossing of a $40-\mathrm{km}$ baseline with speed $v_{t g}=50 \mathrm{~m} / \mathrm{s}$. For instance, for a $0.75-\mathrm{m}$ wavelength, the maximum coherent integration time increases from 0.16 in MR (aspect

\begin{tabular}{|c|c|c|c|c|c|c|c|c|}
\hline \multirow{4}{*}{$\begin{array}{l}\Delta \varphi / \Delta t \\
(\% / \mathrm{s}) \rightarrow \\
\lambda(\mathrm{m}) \downarrow\end{array}$} & \multicolumn{6}{|c|}{ Monostatic } & \multirow{3}{*}{\multicolumn{2}{|c|}{$\begin{array}{c}\text { FSR } \\
\text { Baseline }=40 \mathrm{~km} \\
v_{t g}=50 \mathrm{~m} / \mathrm{s}\end{array}$}} \\
\hline & \multicolumn{2}{|c|}{0.2} & \multicolumn{2}{|c|}{0.4} & \multicolumn{2}{|c|}{0.8} & & \\
\hline & & & & & & & & \\
\hline & $\Delta f_{M}$ & $\Delta \boldsymbol{\tau}_{M}$ & $\Delta f_{M}$ & $\Delta \boldsymbol{\tau}_{M}$ & $\Delta f_{M}$ & $\Delta \boldsymbol{t}_{M}$ & $\Delta f_{F S}$ & $\Delta \boldsymbol{T}_{F S}$ \\
\hline 3.0 & 0.8 & 1.25 & 1.6 & 0.63 & 3.2 & 0.31 & 0.013 & 75 \\
\hline 1.5 & 1.6 & 0.63 & 3.2 & 0.31 & 6.4 & 0.16 & 0.026 & 37.5 \\
\hline 0.75 & 3.2 & 0.31 & 6.4 & 0.16 & 12.8 & 0.09 & 0.053 & 18.8 \\
\hline 0.3 & 8.0 & 0.13 & 16.0 & 0.06 & 32.0 & 0.03 & 0.13 & 7.5 \\
\hline 0.1 & 24.0 & 0.04 & 48.0 & 0.02 & 96.0 & 0.01 & 0.4 & 2.5 \\
\hline 0.03 & 80.0 & 0.01 & 160.0 & 0.006 & 320.0 & 0.003 & 1.3 & 0.75 \\
\hline
\end{tabular}
angle rate $0.4^{\circ} / \mathrm{s}$ ) to $19 \mathrm{~s}$ in FSR. So, in FSR in addition

Table 3 Maximum coherent integration times for monostatic and FS radar 
to a significant increase of RCS in the FS region, the potential time for coherent integration is also much larger than in MR due to the absence of phase fluctuations.

The very high-frequency resolution of FSR enables development of efficient automatic target classification algorithms based on inverse shadow aperture synthesis and this even allows target profile reconstruction [21,34]. Using the example outlined above we can see that frequency resolution at $0.75-\mathrm{m}$ wavelength with $0.4 \%$ aspect angle rate in Table 3 is $0.05 \mathrm{~Hz}$ for FSR but $\sim 6 \mathrm{~Hz}$ for MR.

\subsection{FS Doppler signature}

Lets assume that the target is moving at an angle $\delta$ relative to the bistatic angle bisector with speed $v_{t g}$ (see Figure 1). Because the FS effect is observed within narrow spatial angles from the baseline, the assumption that target trajectory is linear and speed is constant is accurate for many practical scenarios. The target's bistatic Doppler frequency in this case may be calculated by [27],

$$
f_{D}=\left(2 v_{t g} / \lambda\right) \cos \delta \cos (\beta / 2) .
$$

In the narrow FS region where $\theta_{\mathrm{FS}} \approx K \lambda / D<<1$ as discussed in Section 2.2, the FS Doppler frequency $f_{D \text {, FS }}$ is described by

$$
\begin{aligned}
f_{D, \mathrm{FS}} & \leq\left(2 v_{t g} / \lambda\right) \cos \left(\frac{\pi}{2}-\frac{K \lambda}{4 D}\right) \cos (\delta) \\
& =\left(2 v_{t g} / \lambda\right) \sin \left(\frac{K \lambda}{4 D}\right) \cos (\delta) \\
& \approx K \frac{2 v_{t g}}{\lambda} \cdot \frac{\lambda}{4 D} \cos (\delta) \approx \frac{K v_{t g} \cos (\delta)}{2 D} .
\end{aligned}
$$

It should be stressed that in the inequality (11), dependence on the carrier is absent which allows estimation of the Doppler frequency shift within the FSML using only the target parameters of trajectory, speed and size. Obviously, the maximum Doppler shift corresponds to the target trajectory perpendicular to the baseline (i.e. when $\delta=0^{\circ}$ ) and therefore, within the FSML, it is specified only by target speed and effective target dimension. The larger the target effective dimension, the lower the Doppler shift at the edge of the FSML of such a target for fixed speed. To illustrate such invariance of the Doppler shift within the FSML in relation to carrier frequency (or rather to the electrical size of the target), three measured Doppler signatures with highlighted sections of signal corresponding to the FSML are shown in Figure 7, for a car (Land Rover Discovery 2), length $4.5 \mathrm{~m}$, height 1.9 crossing the middle of a $50-\mathrm{m}$ baseline with speed $v_{t g}=5.5 \mathrm{~m} / \mathrm{s}$, at frequencies of $135 \mathrm{MHz}$ (a) and $434 \mathrm{MHz}$ (b) and an engine powered inflatable boat crossing the middle of a 350-m baseline, length $2.3 \mathrm{~m}$, $v_{t g}=5.0 \mathrm{~m} / \mathrm{s}$ at a frequency of $7.5 \mathrm{GHz}(\mathrm{C})$. In these figures, the highlighted section of signature was extracted using a procedure based on coherent signal processing of the Doppler signature as described in [22]. Initially, optimal filtering is performed, where the measured signature is correlated against a set of pre-defined reference functions. Once the maximum correlation and therefore the matching reference waveform is found (Figure 8a,b), this allows extraction of the speed and trajectory of the target which are then used to estimate the RCS envelope of the target in the time domain. The Doppler frequency evaluated at the nulls defining the main lobe of the FSCS in the time domain (Figure 8b, shaded area) gives the maximum of the Doppler frequency shift within the FSML. The power spectral densities (PSDs) of the measured signals of Figure $7 \mathrm{a}-\mathrm{c}$ truncated inside the FSML are calculated and shown in Figure $7 d-f$, respectively. The electrical sizes of the targets and, therefore, scattering mechanisms differ significantly for all three cases. At a frequency of $135 \mathrm{MHz}$ (2.22-m wavelength) the scattering mechanism for a medium size vehicle corresponds to the Mie-Rayleigh region boundary, at $434 \mathrm{MHz}(69 \mathrm{~cm})$ it is sub-optical and the boat illuminated at $7.5 \mathrm{GHz}$ (4 cm wavelength) is in the optical scattering region. However, the Doppler spectra for all the cases are less than $8 \mathrm{~Hz}$ (at the $-10 \mathrm{~dB}$ level) for the range of targets of interest, although the carrier frequencies differ approximately 50 times.

Thus, the Doppler shift observed within the FSML is small and for most practical cases is in the order of a few Hz. In the case of traditional BR and PCL systems, this area of low Doppler would be referred to as a dead zone and excluded from the analysis-special techniques should be applied to detect these signals, as will be discussed. Such a low Doppler frequency spectrum of the main energy part of the signal requires an analysis of the radar sensitivity and, in particular, the effect of the phase noise of the transmitter on FSR performance.

\section{Practical Realisation of FSR}

Here we look at the actual system design though consideration of power budget, phase noise and ultimately the hardware required in order to realise the system physically and that allows it to function in the manner shown in previous sections.

\subsection{Power budget and phase noise}

In FSR, the signal scattered from a target is received as a modulation on top of the direct path (leakage) signal and is consequently subject to leakage signal phase noise. Lets first evaluate the target signal-to-leakage ratio (SLR) in FSR. 

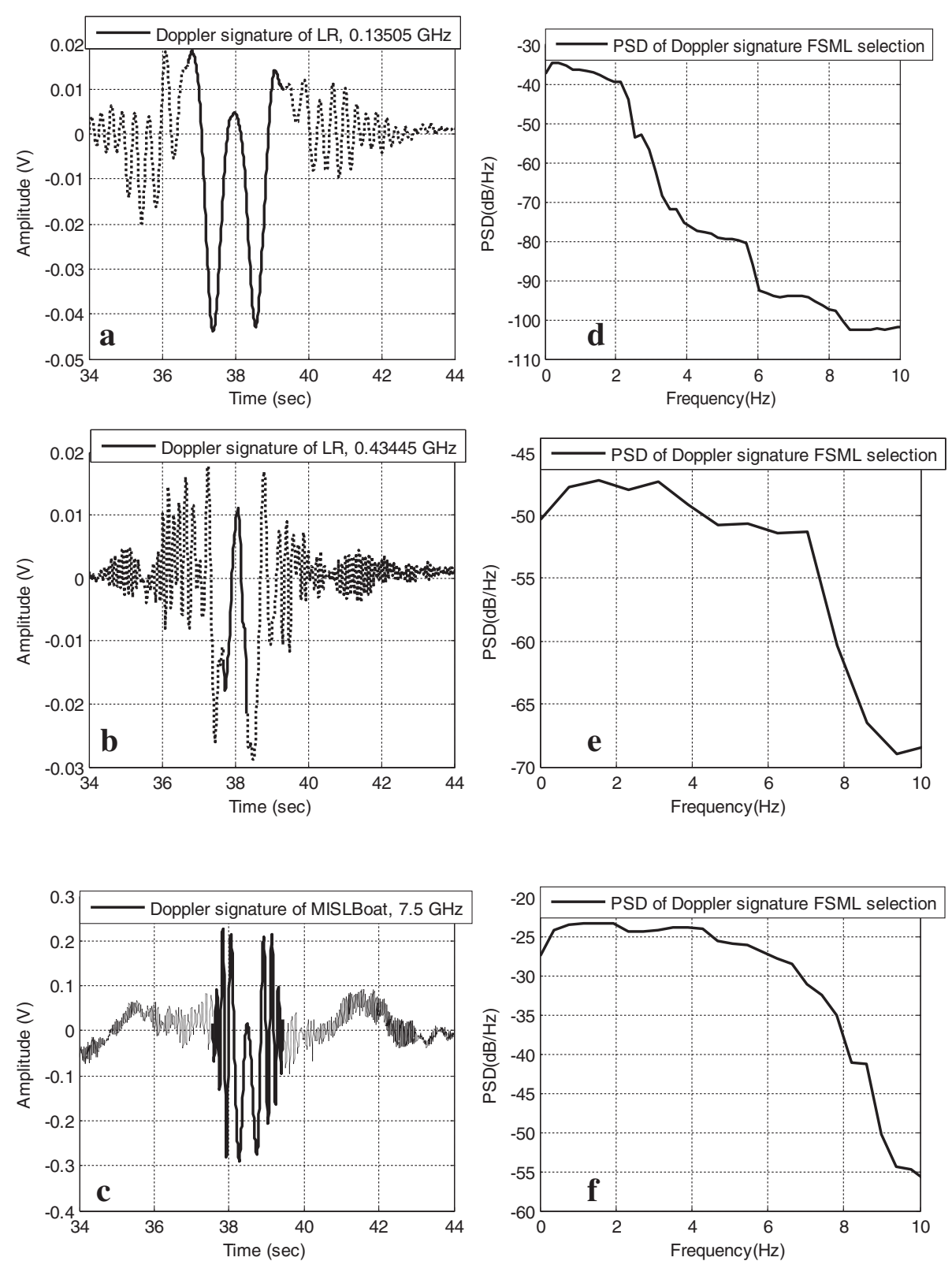

Figure 7 FSR Signatures of targets at three frequencies. Land Rover signature at $135 \mathrm{MHz}(\mathbf{a})$ and at $434 \mathrm{MHz}(\mathbf{b})$; inflatable boat signature at $7.5 \mathrm{GHz}(\mathbf{c})$ and corresponding FS PSD (d-f).

The received leakage signal power in the free space model is

$$
P_{L}=\frac{P_{A \nu} G_{T} G_{R} \lambda^{2}}{(4 \pi)^{2} R_{0}^{2} L_{1}}
$$

while the maximum level of received scattered target signal in the FSML [17] is

$$
P_{\mathrm{Rx}} \approx \frac{P_{\mathrm{Av}} G_{T} G_{R} \lambda^{2} \sigma_{\mathrm{Max}}}{(4 \pi)^{3} R_{T}^{2} R_{R}^{2} L_{0}},
$$

where $P_{\mathrm{Av}}$ is the transmitting average power, $G_{T}$ and $G_{R}$ are the transmit and receive antenna gains, $R_{T}$ and $R_{R}$ are the target to transmitter/receiver ranges, respectively, $\sigma_{\operatorname{Max}}$ is the maximum FSCS and $L_{0}$ and $L_{1}$ are extra losses. Thus, the SLR is:

$$
S L R \approx \frac{R_{0}^{2} \sigma_{M a x} L_{1}}{4 \pi R_{T}^{2} R_{R}^{2} L_{0}} .
$$

Under other equal conditions the minimum of (14) is obtained if $R_{T}=R_{R}=R_{0} / 2$, i.e. when a target is crossing the middle of the baseline, thus: 

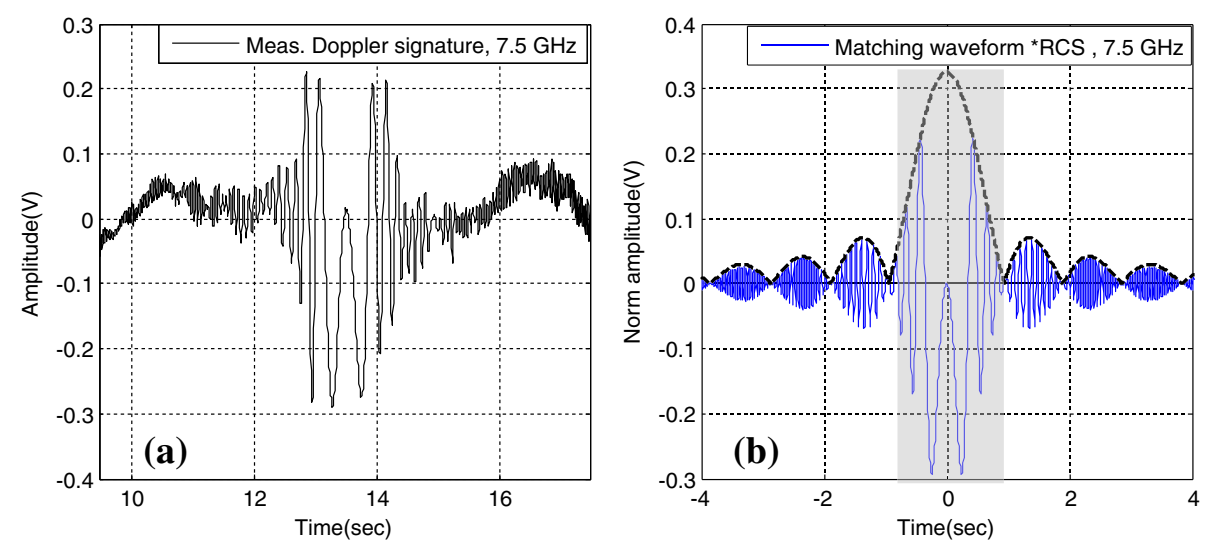

Figure 8 Determination of the time domain signal section corresponding to the FSML. The initial Doppler signature (a) is processed to find a matching waveform (b) and, therefore, the target motion parameters. These are used to form the RCS envelope (dashed line) and the FSML section of the simulated signal is defined (shaded area). Finally, the maximum FS Doppler frequency is evaluated at the edge of FSML section.

$$
\mathrm{SLR}_{\mathrm{Min}}=\frac{4 \sigma_{\mathrm{Max}} L_{1}}{\pi R_{0}^{2} L_{0}}
$$

Then in the first approximation $L_{1} \approx L_{0}$ and

$$
\mathrm{SLR}_{\mathrm{Min}, \text { Free }}=\frac{4 \sigma_{\mathrm{Max}}}{\pi R_{0}^{2}}
$$

This model perhaps is not typical for PCL and specifically for those operating in VHF/UHF bands, e.g. Radio and TV broadcasting, where both transmit and receive antenna beams are touching the surface and reflection from the ground must be taken into account. The free space model may better serve at L-band and higher operational frequencies, although even in this case, the model (14) is only applicable for air target detection. For low-frequency PCL and/or ground targets, the two-ray path (TRP) propagation model must be exploited and assuming an ideally conducting ground surface it gives

$$
P_{L}=P_{\mathrm{AV}} G_{T} G_{R} \frac{h_{T}^{2} h_{R}^{2}}{R_{0}^{4}},
$$

thus

$$
\begin{gathered}
P_{\mathrm{Rx}, \mathrm{Min}}=P_{\mathrm{AV}} G_{T} G_{R} \cdot \frac{4 \pi \sigma_{\mathrm{Max}}}{\lambda^{2}} \frac{h_{T}^{2}}{R_{0}^{4} / 2^{4}} \cdot \frac{h_{T g}^{2}}{R_{0}^{4} / 2^{4}} \\
=P_{\mathrm{AV}} G_{T} G_{R} \cdot \frac{2^{10} \pi \sigma_{\mathrm{Max}}}{\lambda^{2}} \frac{h_{T}^{2} h_{T g}^{2}}{R_{0}^{4}} \cdot \frac{h_{T g}^{2} h_{R}^{2}}{R_{0}^{4}},
\end{gathered}
$$

and

$$
\mathrm{SLR}_{\mathrm{Min}, \mathrm{TRP}}=\frac{1024 \cdot \pi \sigma_{\mathrm{Max}}}{\lambda^{2}} \frac{h_{T g}^{4}}{R_{0}^{4}} .
$$

where $h_{T}$ and $h_{R}$ are the transmit and receive antenna elevations, respectively, and $h_{T g}$ is either the air target altitude or effective height of the surface target, which in the first approximation may be considered as the half of the target height $[17,23]$.
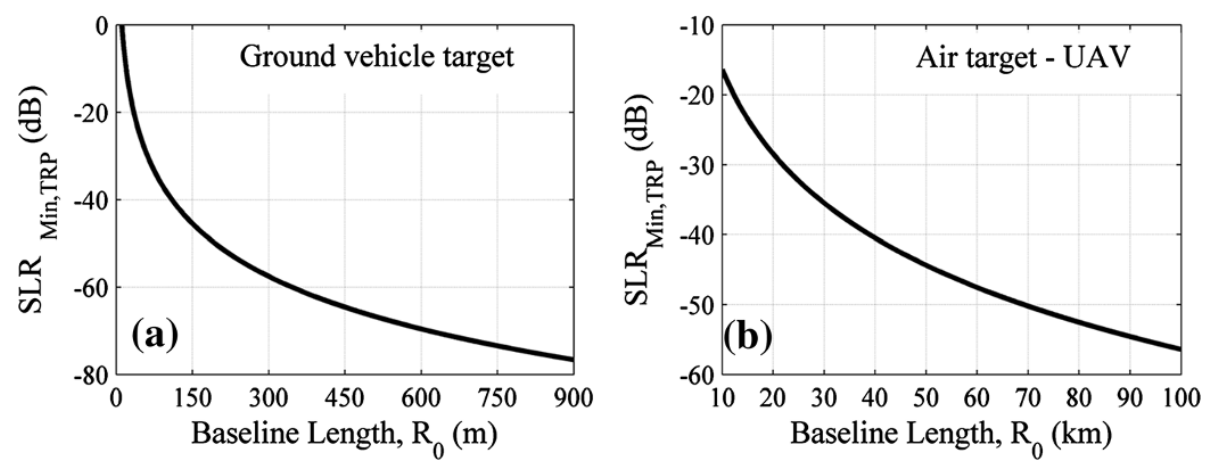

Figure 9 SLR calculated by TRP model of car (a) and UAV (b). Dimensions of targets correspond to those presented in Table 2. 


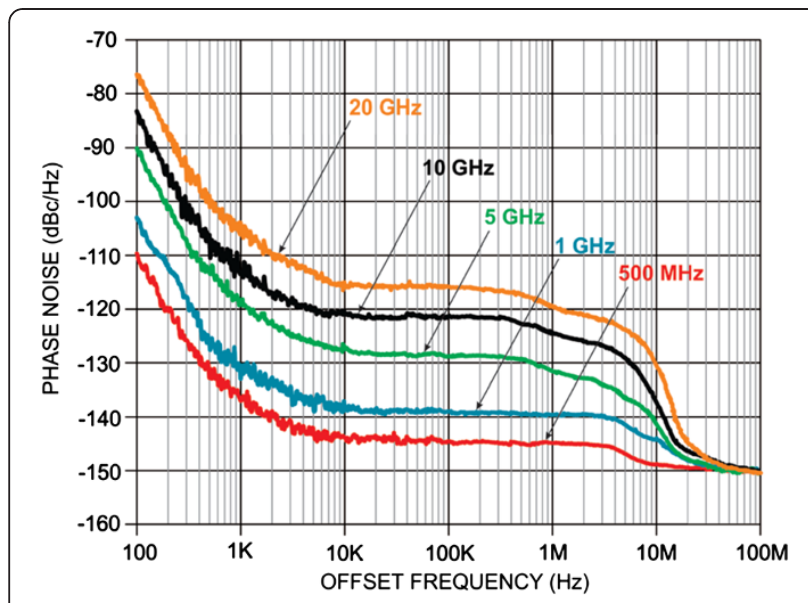

Figure 10 Typical phase noise level in modern synthesisers. Plot is taken from PhaseMatrix, Inc., web-site [36].

In Figure 9, the calculated minimum SLR in the TRP

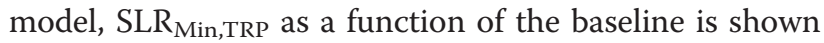
for a $\operatorname{DAB}(\lambda=1.5 \mathrm{~m})$ PCL for both a ground vehicle (with an effective height $h_{T g}=1 \mathrm{~m}$ and $\sigma_{\mathrm{Max}}=10 \mathrm{dBsm}$ ) and air target (with $\sigma_{\mathrm{Max}}=20 \mathrm{dBsm}$ flying at $200-\mathrm{m}$ altitude). From this figure, it is seen that the SLR can be as small as -60 to $-80 \mathrm{~dB}$. The measured SLR from a car (Land Rover Discovery) at the same frequency with a $300-\mathrm{m}$ baseline is about $-70 \mathrm{~dB}$ which reasonably well corresponds to value of $-58 \mathrm{~dB}$ calculated by Equation (19).

The next step is to estimate the leakage signal phase noise level. The absolute noise level depends on a particular frequency synthesiser's quality. In Figure 10, the typical phase noise levels at the synthesiser output are shown for various carrier frequencies. For the expected FSR Doppler frequency range, which is below $10 \mathrm{~Hz}$ (Table 3), curves in Figure 10 can be extrapolated back towards the carrier (continuing the slope at $-30 \mathrm{~dB}$ per decade) so that, for the frequency offset of $1-10 \mathrm{~Hz}$, we can expect the phase noise level to be between -20 and $-50 \mathrm{dBc} / \mathrm{Hz}$ for $20 \mathrm{GHz}$ and -60 and $-90 \mathrm{dBc} / \mathrm{Hz}$ at $500 \mathrm{MHz}$. It has also been reported that the phase noise in terrestrial DAB for the offset $1-10 \mathrm{~Hz}$ is approximately $-50 \mathrm{dBc} / \mathrm{Hz}$ [35]. Consequently, BR in FS mode will be limited by the transmitter phase noise. A possible solution is to extract the signal by means of a selfmixing receiver, i.e. the receiver where the leakage signal is acting as a heterodyne to the FS target signal. The two signals have near zero relative delay due to the very close vicinity of the target to the baseline and in this case the leakage phase noise will be converted to DC at the mixer output.

\subsection{Self mixing receiver for PCL FS operational mode}

As long as the leakage signal is above the thermal noise of the radar receiver, any nonlinear component could be used as the mixer. Two approaches may be recommended: firstly, an envelope detector with quadrature nonlinear characteristic and secondly, the received signal strength indicator output of amplifiers contained in off-the-shelf chipsets.

Here, we consider the basic relationships between input and output signals in a quadrature detector. The simplified FS receiver block diagram is shown in Figure 11 (see also Figure 1). The nonlinear element has the transfer characteristic $S_{\text {out }}=\left(S_{\text {in }}\right)^{2}$, and there are two signals present at its input, the leakage signal (phase noise free in this example) and a signal scattered from a target with Doppler shift $\omega_{d}$ [19]. At the output of the nonlinear element, the signal will be

$$
S_{\text {out }}(t) \approx\left[A_{\mathrm{DPS}} \cos \left(\omega_{0} t\right)+A_{T g} \sin \left(\left(\omega_{0}+\omega_{d}\right) t\right)\right]^{2},
$$

where $A_{\mathrm{DPS}}$ and $A_{T g}$ are the amplitudes of the direct path signal (leakage) and target scattered signal, respectively. After low pass filtering, the signal will contain only two spectral components-DC from the direct path signal and Doppler from the target

$$
S_{\text {out }}(t) \approx \Theta\left(\left(A_{\mathrm{DPS}}^{2}+A_{T g}{ }^{2}\right)+A_{\mathrm{DPS}} A_{T g} \sin \left(\omega_{d} t\right)\right),
$$

where $\Theta$ is the conversion coefficient. The actual value of the conversion coefficient $\Theta$ depends on the type of nonlinear device. Taking into account that the leakage signal is much stronger than the target signal, the leakage may act as the pumping waveform and the nonlinear element is acting as a parametric mixer.

Figure 12 shows the results of a laboratory experiment to measure very low Doppler frequencies from a slowly rotating three-blade propeller, at a carrier frequency of $7.5 \mathrm{GHz}$ in the FSR configuration. The figure indicates that the system is capable of measuring Doppler frequencies as low as

Antenna

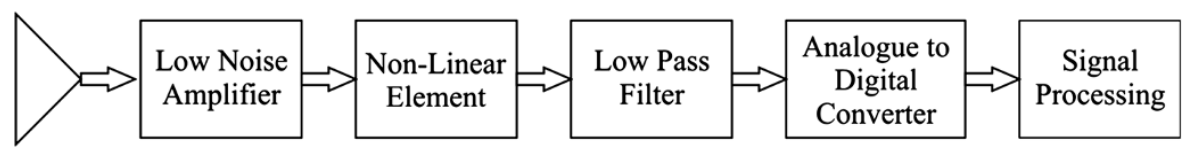

Figure 11 FSR channel block diagram. 


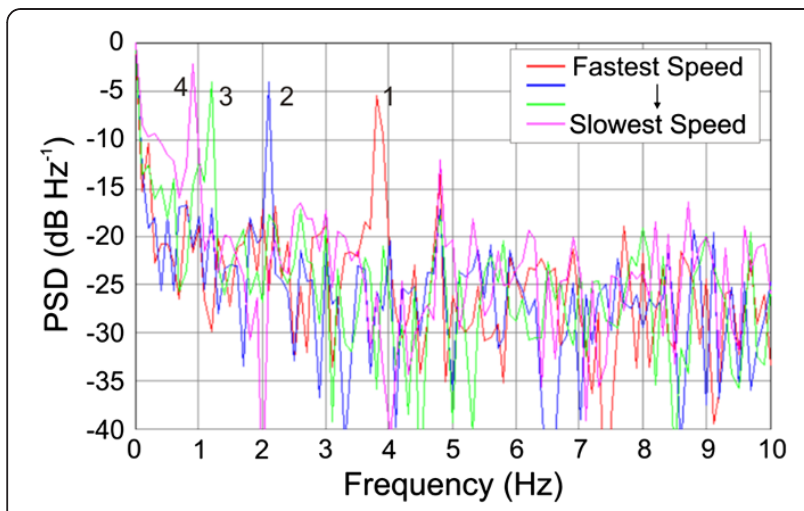

Figure 12 Doppler signature of three-blade propeller measured at four different rotation speeds. Peaks indicate Doppler frequency of the rotating target for four speeds, ranging between peak 1, the fastest (200 rpm) and the slowest, peak 4 (60 rpm).

$1 \mathrm{~Hz}$. This is precisely due to the FS radar system topology, which uses the direct signal from the transmitter as a pseudo local oscillator to mix with the received target FS signal to produce the Doppler output. Any noise of the transmitter (which would end up as a phase noise in a traditional backscatter radar receiver and produce low-frequency spectral components in any subsequent processing) will correlate with that in the target scattered signal and thus will ultimately not be seen as a significant noise component. In all cases of self-mixing hardware signal processing, the phase noise is converted to the DC component of the output. Furthermore, the self-mixing procedure removes any modulation of the transmitting signal, making signal processing in PCL independent of the specific modulation of the transmitter. Figure 13 shows the result of an experiment conducted in order to demonstrate this independence. Two FSR Doppler signatures of a small inflatable boat crossing a 300-m baseline were measured using (a) a non-modulated continuous wave $7.5-\mathrm{GHz}$ signal, and then (b) an UWB signal of 3-GHz bandwidth centred at 7.5 GHz. Qualitatively the signals, recorded successively, appear very similar, demonstrating that even UWB modulation of the transmitted signal does not affect the FS target signature. The slight difference in the waveforms is related to presence of the dynamic sea clutter background

Thus, utilisation of self-mixing receiver gives two main advantages:

1. It removes the phase noise and modulation of the transmitter in FS PCL which makes signals detectable even at nearly zero Doppler shifts.

2. In addition, removing the transmitter modulation leads to the simple and universal signal processing algorithms for all FS PCL systems, irrelevant of the transmitted waveforms.

\section{Conclusions}

PCL has been discussed previously in BR configuration. The transfer of forward scatter concept to PCL systems (FS PCL) provides a new emerging area of research. PCL can naturally use its network structure of transmitters of opportunity, e.g. TV and DAB broadcasting and cellular radio networks, various GNSS systems, which makes FS PCL even more attractive in comparison with a single or chained dedicated FSR.

In this article, we have analysed the performance of the FSR in relation to PCL on its ability to deliver information on 'difficult' targets, have shown its advantages such as enhanced cross section in forward direction, Doppler resolution and utilising the leakage signal as a pseudo local oscillator to mix with the received target FS signal to produce the Doppler output. We also have scrutinised the FS signals in view of finding key effects and parameters influencing the waveforms and spectra which define the overall signal processing.
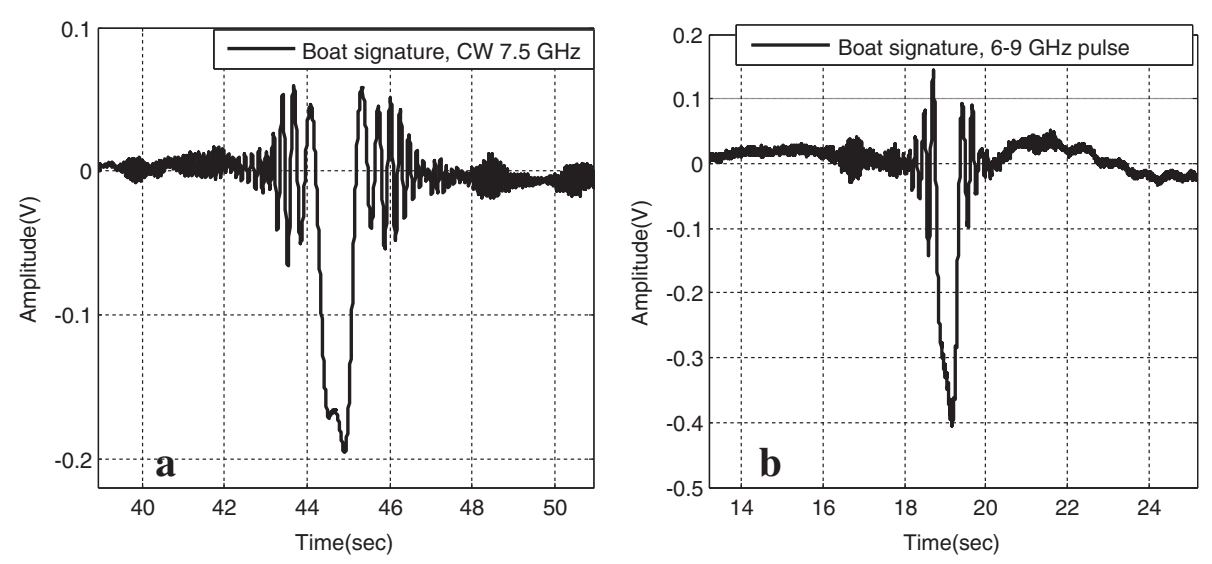

Figure 13 FS signature of a boat obtained by modulated and non-modulated signals. (a) For the non-modulated CW signal and (b) for an UWB-3-GHz bandwidth modulated signal. 
The region in the vicinity of the baseline has been considered in many cases as a 'dead zone' due to the very low Doppler frequency of the moving target in this region. Utilising a relatively simple modification in signal processing at hard and/or software levels, this region can be considered as operational region for FS PCL with enhanced target detection and automatic target recognition capabilities. Also it is worthwhile noting that due to self-mixing receiver architecture in FS mode the modulation of the specific PCL transmitter of opportunity does not influence the processing of the signals. Consequently, an FS PCL network can be formed using a multitude of signals from various transmitters of opportunity.

The PCL operating in VHF/UHF bands can particularly be recommended for airborne target detection in the FS region, while for the surface targets practically all PCL sources could be used.

\section{Competing interests}

The authors declare that they have no competing interests.

\section{Author details}

${ }^{1}$ Electronic, Electrical \& Computer Engineering, University of Birmingham, Edgbaston, Birmingham B15 2TT, UK. ${ }^{2}$ The National Research University of Electronic Technology, Moscow 124498, Russia.

Received: 30 July 2012 Accepted: 5 February 2013

Published: 27 February 2013

\section{References}

1. NJ Willis, HD Griffiths, Advances in Bistatic Radar (SciTech Publishing, Raleigh, NC, 2007)

2. M Cherniakov (ed.), Bistatic Radar: principles and practice (John Wiley \& Sons, 2007)

3. H Kuschel, D O'Hagan, Passive radar from history to future (in 11th International Radar Symposium (IRS) 2010, Vilnius, Lithuania, 2010), pp. 1-4. 16-18 June

4. PE Howland, D Maksimuk, G Reitsma, FM radio based bistatic radar. IEE Proceedings - Radar, Sonar and Navigation 152(3), 107-115 (2005)

5. D Poullin, M Flecheux, Recent progress in Passive Coherent Location (PCL) concepts and technique in France using DAB or FM broadcasters, in Proc. of IEEE Radar Conference RADAR'08, Rome, Italy, 2008, pp. 1-5

6. R Saini, M Cherniakov, DTV signal ambiguity function analysis for radar application. IEE Proceedings - Radar, Sonar and Navigation 152(3), 133-142 (2005)

7. M Cherniakov, D Nezlin, K Kubik, Air target detection via bistatic radar based on LEOs communication signals. IEE Proceedings - Radar, Sonar and Navigation 149(1), 33-38 (2002)

8. DKP Tan, H Sun, Y Lu, M Lesturgie, HL Chan, Passive radar using Global System for Mobile communication signal: theory, implementation and measurements. IEE Proceedings - Radar, Sonar and Navigation 152(3), 116-123 (2005)

9. F Colone, K Woodbridge, H Guo, D Mason, CJ Baker, Ambiguity Function Analysis of Wireless LAN Transmissions for Passive Radar. IEEE Trans. Aerosp. Electron. Syst. 47(1), 240-264 (2011)

10. H Guo, K Woodbridge, CJ Baker, Evaluation of WiFi beacon transmissions for wireless based passive radar, in Proc. of IEEE Radar Conference RADAR'08, Rome, Italy, 2008, pp. 1-6

11. R Saini, Z Rui, M Cherniakov, Development of space-surface bistatic synthetic aperture radar with GNSS transmitter of opportunity, in Proc. of IEEE Radar Conference RADAR'08, Rome, Italy, 2008, pp. 1-6

12. M Cherniakov, R Saini, R Zuo, M Antoniou, Space surface bistatic SAR with space-borne non-cooperative transmitters, in European Rad. Conf. EURAD2005, Paris, France, 2005, pp. 9-12

13. I Walterscheid, T Espeter, AR Brenner, J Klare, JHG Ender, H Nies, R Wang, O Loffeld, Bistatic SAR Experiments With PAMIR and TerraSAR-X_-Setup,
Processing, and Image Results. IEEE Trans Geosci Rem Sens 48(8), 3268-3279 (2010)

14. L Cazzani, C Colesanti, D Leva, G Nesti, C Prati, F Rocca, D Tarchi, A GroundBased, Parasitic SAR Experiment. IEEE Trans Geosci Rem Sens 38(5), 2132-2141 (2000)

15. HD Griffiths, CJ Baker, Passive coherent location radar systems. Part 1: performance prediction. IET Radar, Sonar \& Navigation 152(3), 153-159 (2005)

16. CJ Baker, HD Griffiths, I Papoutsis, Passive coherent location radar systems. Part 2: waveform properties. IET Radar, Sonar \& Navigation 152(3), 160-168 (2005)

17. V Sizov, M Cherniakov, M Antoniou, Forward Scattering Radar Power Budget Analysis for Ground Targets. IET Radar, Sonar \& Navigation 1(6), 437-446 (2007)

18. M Cherniakov, RSAR Abdullah, P Jancovic, M Salous, V Chapursky, Automatic ground target classification using forward scattering radar. IEE Proceedings on Radar, Sonar and Navigation 153(5), 427-437 (2006)

19. M Gashinova, L Daniel, K Kabakchiev, V Sizov, E Hoare, M Cherniakov, Phenomenology of signals in FSR for surface targets detection, in International Conference on Radar Systems RADAR 2012 (, Glasgow, UK, 2012), p. 6

20. M Cherniakov, M Gashinova, H Cheng, M Antoniou, V Sizov, LY Daniel, Ultra wideband forward scattering radar: Concept and prospective, in 2007 IET International Conference on Radar Systems, Edinburgh, UK, 2007, pp. 1-5

21. W Chapurskiy, VN Sablin, SISAR: Shadow Inverse synthetic aperture radiolocation, in Proc. of the IEEE-Radar 2000 International Conference, Alexandria, VA, USA, 2000), pp. 322-328

22. H Cheng, V Sizov, M Antoniou, M Gashinova, M Cherniakov, Optimal Signal Processing in Ground-Based Forward Scatter Micro Radars. IEEE Trans. Aerosp. Electron. Syst. 48(4), 3006-3026 (2012)

23. K Kabakchiev, LY Daniel, V Sizov, E Hoare, M Gashinova, M Cherniakov, Received signal characterization in forward scatter radar for maritime application, in Proceedings of International Radar Symposium 2011 (IRS) Leipzig, Germany, 2011), pp. 67-72

24. P Ufimtsev, New Insight into the Classical Macdonald Physical Optics Approximation. IEEE Antenn Propag Mag 50(3), 11-20 (2008)

25. PY Ufimtsev, Fundamentals of the Physical theory of Diffraction (John Wiley and Sons, 2007)

26. PE Howland, HD Griffiths, CJ Baker, Passive bistatic radar systems, in Bistatic Radar: Emerging Technology, ed. by M Cherniakov (John Wiley \& Sons, 2008), p. 394

27. N Willis, Bistatic Radar (SciTech Publishing, 2007)

28. L Daniel, M Gashinova, M Cherniakov, Maritime Target Cross Section Estimation for an UWB FSR Network, in Proc. EURAD 2008 (, Amsterdam, Netherlands, 2008), pp. 316-320

29. J Glaser, Forward Scatter Radar for Future Systems. The WSTIAC Quarterly 10(3), 3-8 (2011)

30. Ml Skolnik, Radar Handbook, 2nd edn. (McGraw Hill, 1989)

31. JI Glaser, Bistatic RCS of Complex Objects Near Forward Scatter. IEEE Trans Aero Electron Syst AES-21(1), 70-78 (1985)

32. SW Lee, R Mittra, Fourier transform of a polygonal shape function and its application in electromagnetics. IEEE Trans Antenn Propag 31(1), 99-103 (1983)

33. FE Nathanson, JP Reilly, MN Cohen, Radar Design Principles (McGraw-Hill, 1991)

34. A Myakinkov, A Kuzin, M Gashinova, V Sizov, M Cherniakov, Inverse Forward Scatter SAR, in Proc. of the Institute of acoustics, Int. Conf. on Synthetic Aperture Sonar and Synthetic Aperture Radar 2010 (, Lerici, Italy, 2010), pp. 152-156

35. W Hoeg, T Lauterbach (eds.), Digital Audio Broadcasting: Principles and Applications of DAB, DAB+ and DMB (Wiley-Blackwell, 2009)

36. QuickSyn Datasheet, Microwave frequency synthesizer (Phase Matrix. Inc). accessed July 2012, http://www.phasematrix.com/Spec_Sheets/DS_FSW0010_0020.pdf

doi:10.1186/1687-6180-2013-36

Cite this article as: Gashinova et al: Signal characterisation and processing in the forward scatter mode of bistatic passive coherent location systems. EURASIP Journal on Advances in Signal Processing 2013 2013:36. 